\title{
Hemodynamic Regulation Using Fuzzy Logic
}

\author{
N. Sprunk, A. Mendoza G., \\ A. Knoll \\ Department of Informatics \\ Technische Universität München \\ Germany \\ Email:n.sprunk@tum.de
}

\author{
U. Schreiber, S. Eichhorn, \\ J. Hörer, R. Bauernschmitt \\ Department of Cardiovascular Surgery \\ German Heart Center Munich \\ Technische Universität München \\ Germany
}

\begin{abstract}
After cardiac surgery patients often show a cardiovascular instability, which has to be managed by infusing different vasoactive drugs. This work proposes a closed-loop system to automatically react upon changes in the patients state and trigger infusion of indicated drugs. As input signals mean arterial pressure, cardiac output, central venous pressure and systemic vascular resistance were chosen. The vasoactive drugs used are isosorbide dinitrate, norepinephrine, dopamine and additionally hydroxyethyl starch. The controller itself is designed as a controller system with four internal fuzzy controllers, one for each medication dosage to be calculated. The proposed controller is tested with a simulation environment. The environment is able to simulate a canine cardiovascular system and reactions to different medications. The controller is evaluated for three different diseases, for congestive heart failure, hypotension and hypertension. The fuzzy controller system is able to react adequately in all these test cases in the simulation and regulates the hemodynamic signals back within a range of the desired target values.
\end{abstract}

\section{INTRODUCTION}

After cardiac surgery it is very common that patients develop a hemodynamic instability, such as post-operative hypertension, hypotension or congestive heart failure. That is why patients are kept in the intensive care unit to be able to react on any health degradation at any time. By analyzing the hemodynamic signals in certain intervals, the clinical personal assesses the patients state and can manage to keep the patient's signals, and thus his health, within a desired range by infusing vasoactive drugs. Ensuring a continuous surveillance and regulation of the patients state improves the process of recovery after the cardiac operation.

Manual-Programmable syringe pumps, which allow infusing of medication over a certain time interval, already allow continuous drug application. Also the observation by patient monitoring is running all the time. Only the necessary reassessment of the patients state has still to be done by the personal in the intensive care unit. As they usually have multiple patients to take care of, this task is done only in certain intervalls at the moment. The advantage of an automatic hemodynamic regulation would be a continuously analysis of the patient's state, thus making continuous regulation possible.

A closed-loop system is proposed, which can analyze the patients state and takes over this task from the staff. This can ensure the best hemodynamic management in each situation. The controller is designed to react adequately in common situations, namely congestive heart failure, hypotension and hypertension. It automatically runs and handles these situations autonomously. Thus it gives the clinical personal more time for the treatment of critical patients.

The difficulties in developing automatic regulation of the hemodynamic system are among others, the non-linearities of the complex cardiovascular system and the intra- and inter-patient variability. This paper described the development of a hemodynamic controller based on fuzzy logic and its performance in tests in a simulation environment. It has been evaluated in three different ciritcal situations, which might occur after cardiac surgery.

\section{Overview On Previous Research EFForts}

Hemodynamic control is a very common and every-day task of the clinical personal. For this reason there already has been a considerable amount of research in automatic control of hemodynamic variables with various drugs. Isaka and Sebald [1] give a good overview of the research projects until the early 90s. They reviewed different approaches of control strategies for arterial blood pressure regulation. The different strategies are classified into proportional-integral-derivative (PID) controller, optimal controllers, adaptive controllers and rule-based controllers, including fuzzy and neuronal network control. They came to the conclusion, that the results of various studies looked promising, but that there are still issues in the design of a more robust and adapting controller to ensure patient safety. A couple of years later Held and Roy [2] developed a hemodynamic controller of congestive heart failure (CHF) using fuzzy control. This controller used three different operation states, one to handle critical situations, and two different to take over in noncritical situations. The controller was tested extensively in simulations and also in some dog experiments and showed very good results. Huang and Roy [3] have an approach, which is quite unique, as their controller regards both the pulmonary and the systemic circulation. It also uses a fuzzy logic based controller. It has been verified in a simulation model, where it operated effectively for a wide range of patients. Gao and Er [4] proposed a generalized fuzzy neural network for postsurgical blood pressure regulation, which could also be verified as effective in simulations, but they indicate that further study is necessary. Kashihara [5] used a simple neural network 
to implement a multiple adaptive predictive controller for regulation of hemodynamic variables. One limitation is that only one heart-failure condition is tested. To adapt to various heart diseases, further data from animal experiments needs to be gathered. A more recent research by Kumar et al. [6] tested a fuzzy proportional-derivative (PD) controller, which made use of different mathematical models to simulate the response of the cardiovascular system to vasoactive drugs. The problem in this approach was the selection of appropriate mathematical models. Until today there is no automated regulation system available commercially and the research for a controller, which also can ensure patient safety is still on going.

\section{PROPOSED SYSTEM}

The objective of this work is, to create a closed-loop system for hemodynamic control. To develop a controller, which analyzes and interprets the patients state, the knowledge of the doctors in charge in the intensive care unit is needed. Just as a doctor, the controller should be able to handle various patients. Checking for overstepping a crisp threshold for each signal cannot be sufficient to find adequate treatment for multiple cases. Instead doctors consider multiple signals. Each of them has a range of values, they consider as normal, too low or too high. As fuzzy logic has been specifically developed to incorporate such linguistic statements, that is the reason this controller type was chosen in this study.

\section{A. System Components}

The components, present in the intensive care unit at the moment besides the patient, are the patient monitoring and the syringe pumps, both connected to the patient. The patient monitoring continuously observes and displays various hemodynamic signals. The syringe pumps are operated by the staff to apply continuous medication to the patient. To close the loop, between patient monitoring and syringe pumps, two additional components were developed (figure 1): First the fuzzy controller, which analyses the observed signals and second the medication control, which is used as interface to the syringe pumps.

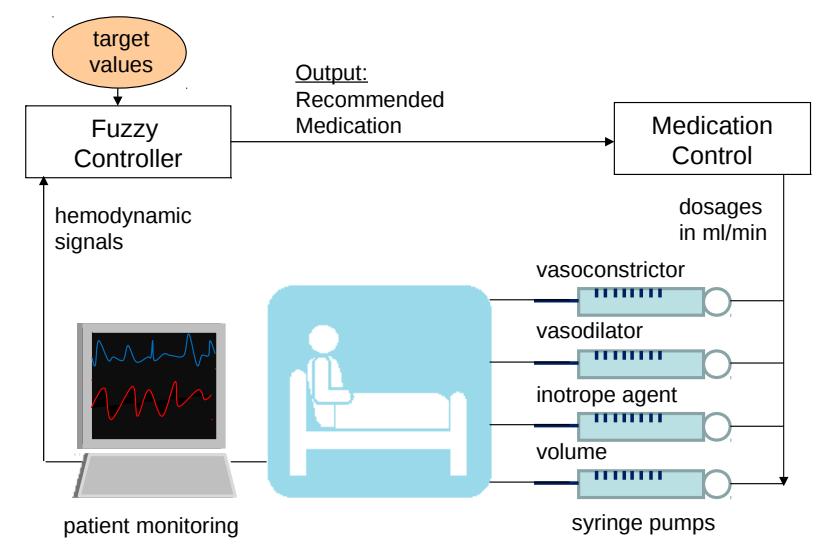

Fig. 1. Closed-loop system with patient and connected hardware devices (patient monitoring and syringe pumps); The fuzzy controller and the medication control close the loop between observation and regulation.
During setup of the controller, a doctor chooses a target value for each hemodynamic signal. In this way the doctor may adapt the controller to inter-patient varieties. For example, in some patients it is desirable to reach a mean arterial pressure (MAP) of $100 \mathrm{mmHg}$, whereas in others $110 \mathrm{mmHg}$ is suitable. After this initial setup, an intervention of a doctor is not needed anymore for hemodynamic regulation. The proposed controller closes the loop from observation to application of the drugs and runs autonomously.

\section{B. Hemodynamic Input Variables}

As hemodynamic variables doctors proposed four inputs, which give them sufficient information to detect the state of the patient and to decide upon the best medication dosage to infuse. These are the inputs chosen also for the fuzzy controller. The first input is the MAP, which is a strong indicator of the workload of the heart and how good the circulation reaches all important organs. If the MAP is too low, the far end of the organs cannot be supplied with enough blood, whereas if MAP is too high the surgical suture lines may rupture resulting in severe bleeding complications. The second parameter to regulate is the Cardiac Output ( $\mathrm{CO}$ ), since it shows how much blood the heart can distribute into the body to keep up a good circulation. In addition to these two, commonly chosen, controlled signals, our controller also takes Central Venous Pressure (CVP) and Systemic Vascular Resistance (SVR) into account. CVP is the pressure in the vena cava, and approximates the pressure in the right atrium of the heart. It is a good indicator for the blood volume in the circulation. The SVR is not measured directly but calculated with formula (1).

$$
S V R=\frac{(M A P-C V P)}{C O}
$$

The SVR is defined by the peripheral circulation and must be overcome by cardiac work load to push blood through the circulatory system.

\section{Medication}

To regulate the hemodynamic system of a patient, there are basically four types of drugs, vasoconstrictors, vasodilators, inotrope agents and volume. Vasocontrictors cause the blood vessel to narrow, whereas vasodilators widen them. In our controller Isosorbide Dinitrate (ISDN) is used as vasodilator to decrease blood pressure. As vasoconstrictor Norepinephrine (NEP) is chosen. Inotrope agents influence the strength of muscles. In hemodynamic regulation positive inotrope agents can be used to increase the contractility of the heart muscle. In intermediate dosages Dopamine (DPM) works as inotrope agent and this was chosen for the proposed controller. Volume increases the total blood volume, so additionally Hydroxyethyl Starch (HES) is suggested by the cardiologists to replace volume in the blood circulation, if the patient is losing total volume. However, this is not tested with the simulation and not analyzed in this paper. 


\section{Fuzzy Controller}

The fuzzy controller system used in our approach has four input variables (MAP, CO, CVP, SVR) and four outputs (ISDN, DPM, NEP, HES). For each output, one internal fuzzy controller is defined. The four controllers have all the same inputs with fuzzy sets named low, normal and high, but they consider different ranges for the membership functions. Their values relate not to the absolute observed value, but to the error calculated between the observed and the target value, which was chosen at setup (figure 2).

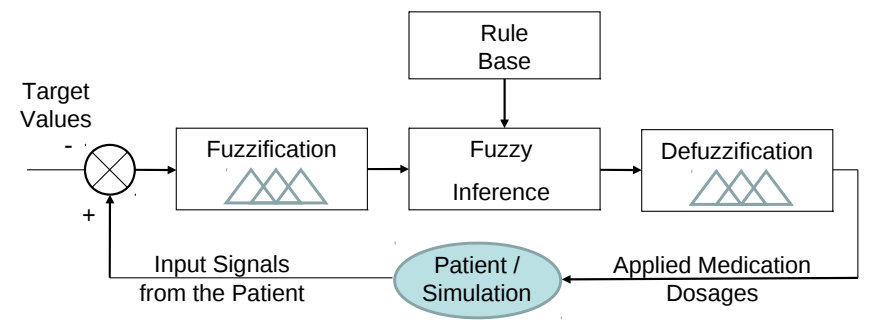

Fig. 2. Design of the Fuzzy Controller; the error between hemodynamic signals observed in patient and their target values, chosen at setup is used as input to the fuzzy controller

The outputs have also each three membership sets (none, medium or high dosage). Again the membership functions are defined with different ranges for each internal controller, as different dosages apply to different drugs.

In figure 3 the used input and output membership functions for the DPM fuzzy controller are shown. The inputs represent the calculated error for each signal, the output is the recommended dosage to react to the patient's state.
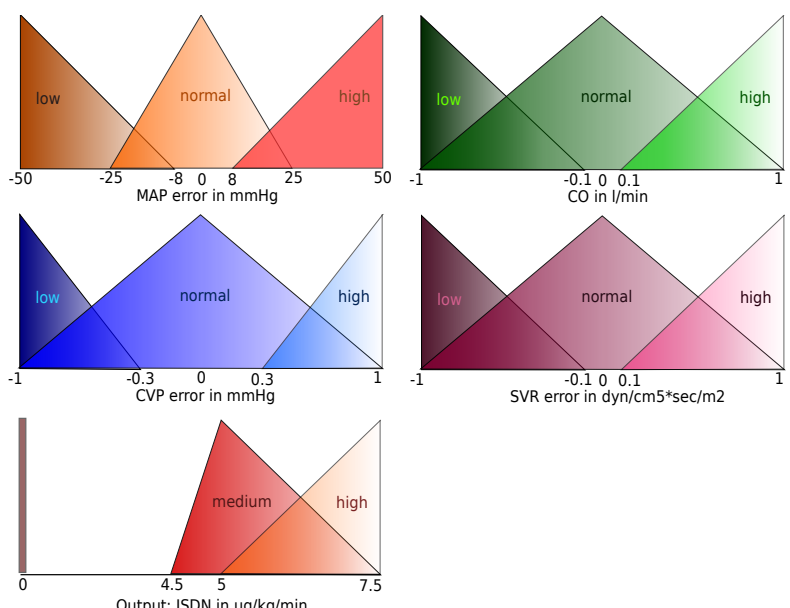

SVR error in dyn/cm $5 * \mathrm{sec} / \mathrm{m} 2$

Fig. 3. Input and output membership functions of internal fuzzy controller for DPM in $\mu \mathrm{g} / \mathrm{kg} / \mathrm{min}$

With four input variables and each with three membership functions, each internal fuzzy controller can have up to 80 unique rules. For each internal fuzzy controller all the possible cases are considered in the rules. As some rules can be combined, the total number of rules is less than 80 for each of the internal controller (see table I).
TABLE I

NUMBER OF RULES DEFINED FOR EACH INTERNAL FUZZY CONTROLLER

\begin{tabular}{|c|c|}
\hline Fuzzy Controller & Number of Rules \\
\hline ISDN & 55 \\
\hline NEP & 25 \\
\hline DPM & 39 \\
\hline HES & 11 \\
\hline
\end{tabular}

In the fuzzification process the crisp error values of each signal is classified in the described membership functions. The fuzzy inference model uses then the defined rules to calculate the output. The result of the calculation done in the Fuzzy Inference component is defuzzyfied using the output membership functions, defined for each drug.

\section{E. Medication Control}

The Medication Control is developed as interface between the fuzzy controller and syringe pumps. The output of the fuzzy controller is a dosage dependent on the patient's weight, for example $5 \mu \mathrm{g} / \mathrm{kg} / \mathrm{min}$. The medication control has the information about the patient's weight and also the concentration of each medication within the syringe pumps. This information is given during setup. With this information the Medication Control calculates the according speed, the syringe pump has to run with. The results are then sent via serial port to each syringe pump. Furthermore the Medication Control also does logging to enable the doctor to see, which medication in which dosage was applied during the controller's operation time.

The implementation for both the Fuzzy Controller and the Medication Control is based on the AutoMedic platform developed by Mendoza et al. [7]. It is a framework, that offers packages for communication, logging or visualization. Based on the components of the Automedic platform our hemodynamic controller was implemented with $\mathrm{C}++$.

\section{F. Simulation Environment}

To test the feasibility of a fuzzy control system in hemodynamic regulation, a simulation was developed based on the work of El-Brawany [8] and Yu et al. [9]. It is implemented in MATLAB and simulates a canine cardiovascular system with 14 compartments. Each compartment is modeled by a nonlinear capacitor, a resistor and an inductance. The model has been enhanced to run in intervals. To send the values of the hemodynamic signals from MATLAB to the $\mathrm{C}++$ application, i.e. the fuzzy controller, the simulation has to be interrupted. The proposed controller runs an analysis every 20 seconds. Thus exactly this time is also simulated and afterwards the simulation is stopped.

Once the controller has calculated the recommended dosages, the Medication Control sends the resulting values to the simulation instead of to the hardware (figure 4). The interface between the fuzzy controller and the Medication Control stay unchanged.

Furthermore the reactions upon infusion of ISDN, NEP and HES were implemented. The simulation model already 


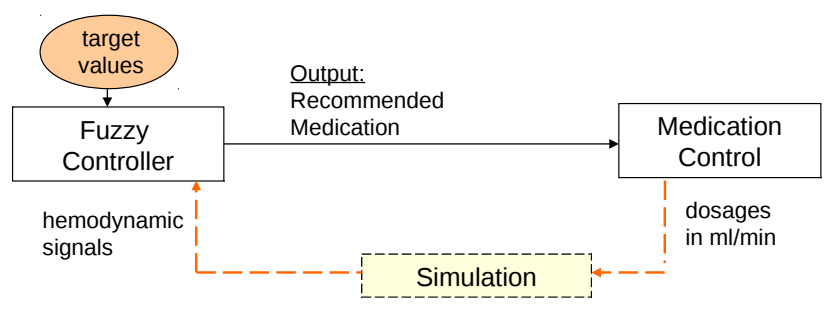

Fig. 4. Simulation loop: The simulation replaces the interface to the patient monitoring and the syringe pumps and simulates the reactions of the patient's cardiovascular system

included an implementation of the reactions to DPM infusion. The reactions to ISDN and NEP were modeled similar to the formulas existent in the model for SNP and DPM respectively, but adapting the parameters to fit the curves of experimental data. The infusion of HES was modeled as an increment of volume in the compartment of systemic veins. The simulation offers the possibility to simulate unhealthy state changes within the patients cardiovascular system. The parameters of the baroreflex component were used to induce hyper- or hypotension. Congestive heart failure (CHF) is simulated by reducing elasticity of the heart. All three disorders can be gradually increased in intensity.

\section{Simulation AND Results}

The proposed fuzzy controller system was verified with the described simulation. Three different test cases were executed, each testing performance in a distinct state of disorder, hypertension, hypotension and congestive heart failure.
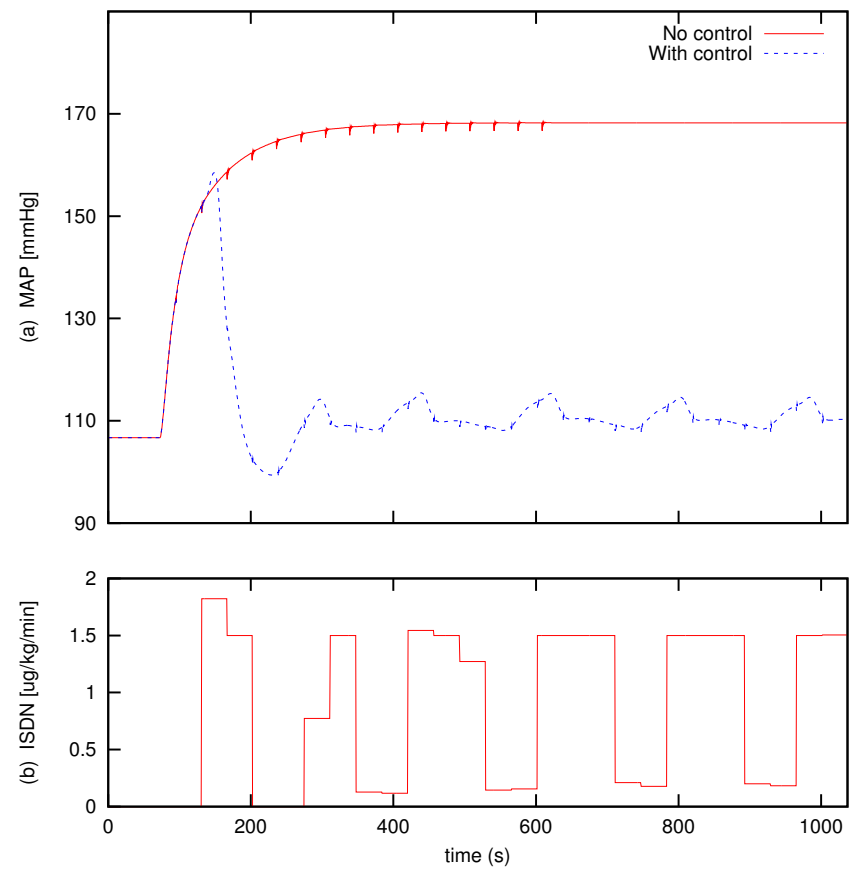

Fig. 5. Test case of hypertension: This shows a comparision of MAP with and without regulation. By infusing displayed ISDN dosage the controller reaches MAP target within $7 \mathrm{mmHg}$.

\section{A. Hypertension}

Through adapting the target of MAP within the baroreflex hypertension is induced in the simulation. Without regulation MAP rises from 105 to $168 \mathrm{mmHg}$ (figure 5a).

When the hemodynamic fuzzy controller is set up with a target value of $105 \mathrm{mmHg}$ for MAP, it automatically applies dosages of up to $1.8 \mu \mathrm{g} / \mathrm{kg} / \mathrm{min}$ ISDN to counteract the hypertension (figure 5c). It starts off with an overshoot of ISDN, causing the MAP to fall under the target value to 99 mmHg. After that the controller manages to keep the MAP within $+7 \mathrm{mmHg}$ of the target value (figure $5 \mathrm{~b}$ ).

\section{B. Hypotension}

When simulating severe hypotension, in the case of no control, MAP falls from 105 down to $73 \mathrm{mmHg}$ (figure 6a). In the same time $\mathrm{CO}$ decreases from 2.05 to $1.7 \mathrm{l} / \mathrm{min}$. If the test is run with active controller and a target value of 105 $\mathrm{mmHg}$ for MAP, it reacts with both a dosage of NEP and also DPM. Both dosages start with a high bolus of $0.05 \mu \mathrm{g} / \mathrm{kg} / \mathrm{min}$ NEP and $3.5 \mu \mathrm{g} / \mathrm{kg} / \mathrm{min}$ of DPM and afterwards decrease as the patient's state improves. These actions of the controller manage to keep the $\mathrm{CO}$ above $21 / \mathrm{min}$ as desired and the MAP within $8 \mathrm{mmHg}$ of its target value (figure $5 \mathrm{~b}$ ).
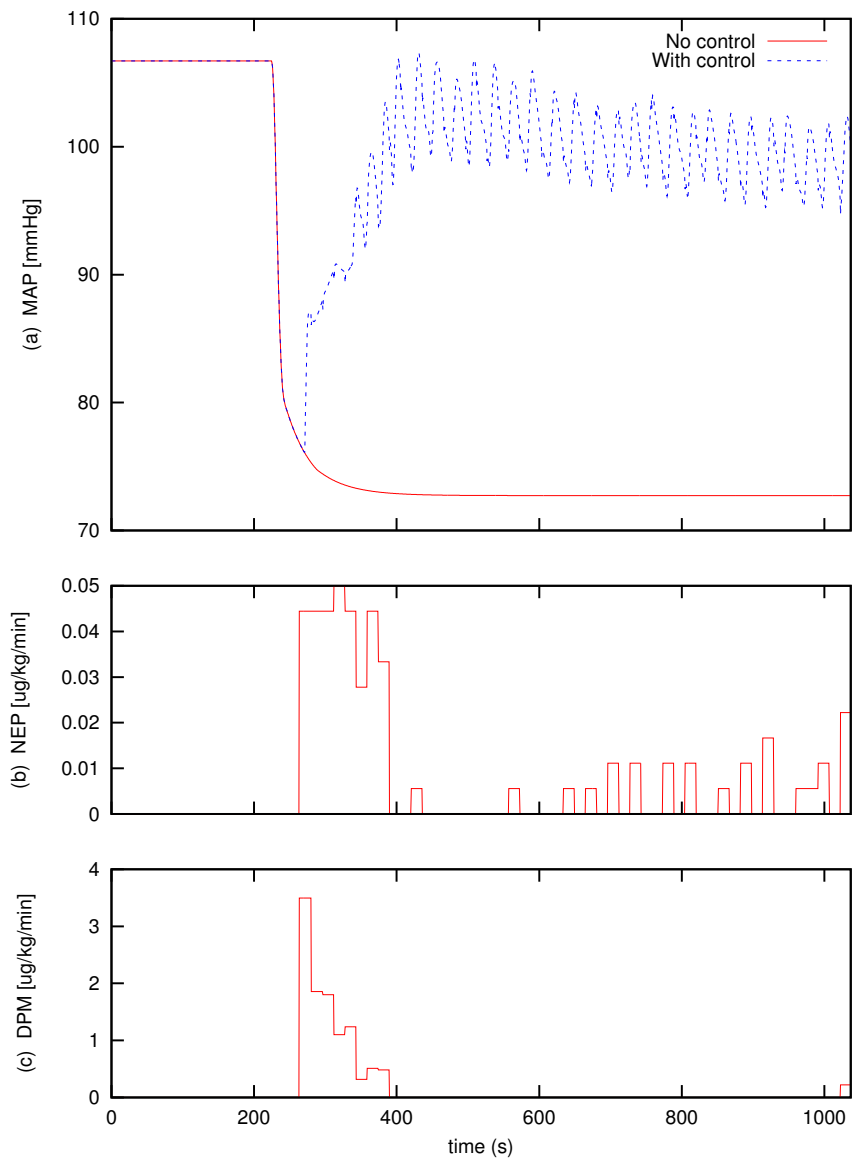

Fig. 6. Test case of hypotension: This shows a comparision of MAP with and without regulation. By infusing displayed NEP and DPM dosages the controller reaches MAP target within $8 \mathrm{mmHg}$. 


\section{CHF with Hypotension}

A very severe condition in patients is CHF combined with hypotension. The MAP decreases to $60 \mathrm{mmHg}$ (figure 7a) and $\mathrm{CO}$ is reduced to $1.3 \mathrm{l} / \mathrm{min}$ in the simulation. The test is run again with the controller active and a target value of $105 \mathrm{mmHg}$ for MAP.
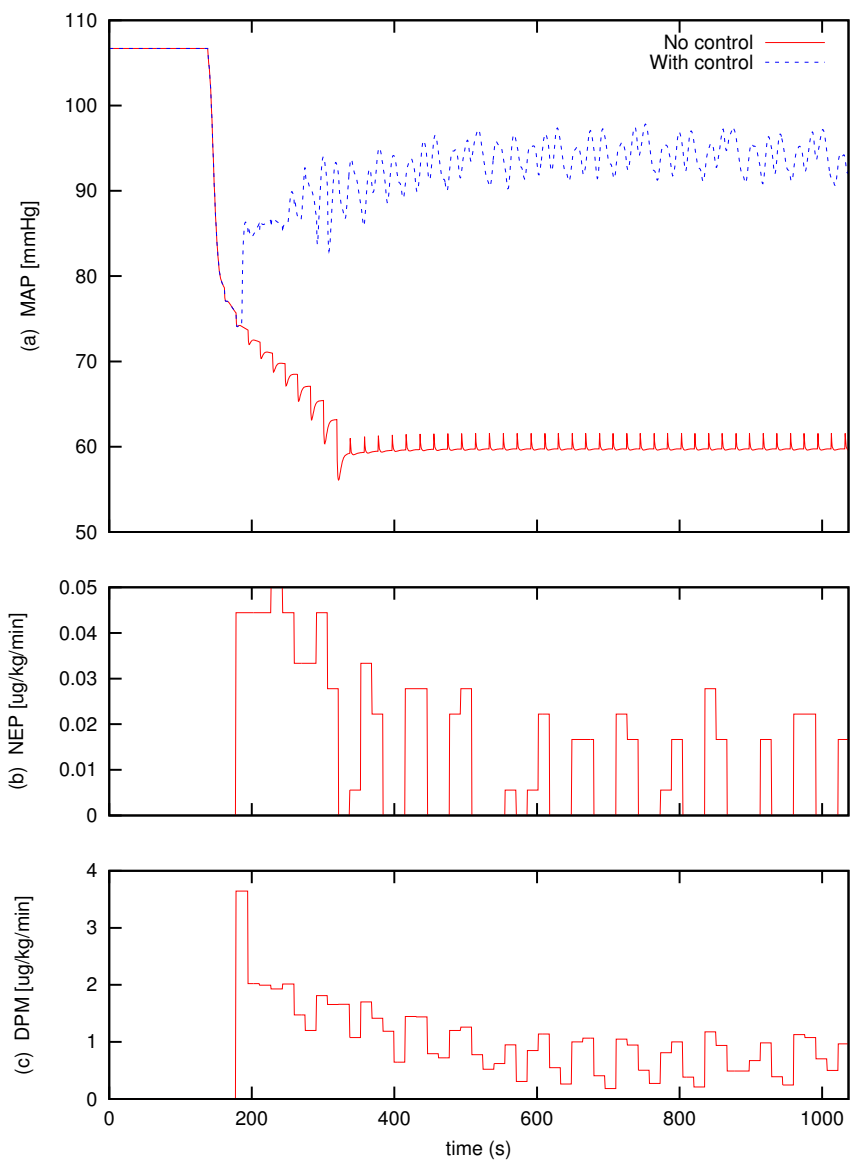

Fig. 7. Test case of congestive heart failure: This shows a comparision of MAP with and without regulation. By infusing displayed NEP and DPM dosages the controller reaches MAP target within $11 \mathrm{mmHg}$.

To regain the contractility of the heart muscle the hemodynamic controller applies DPM (figure 7d) in this situation and NEP (figure 7c) to increase MAP. In this way MAP is again in an area of $11 \mathrm{mmHg}$ of the target value (figure $7 \mathrm{~b}$ ) and $\mathrm{CO}$ is near $2 \mathrm{l} / \mathrm{min}$ again.

\section{Discussion and Future Work}

The simulation results show that the proposed fuzzy controller is applicable in hemodynamic control. The controller manages to keep the hemodynamic signals within a range of the set target values after some time in every case. The continuous regulation ensures a constant adaptation of the medication to the current state.

Still the results also propose that there is some room for improvements. The hypertension test cases shows, that the controller reacts to the high MAP value very fast, but also induces an overshoot. This overshoot is neither necessary nor desireable and the controller needs to be adapted, so this overshoot does not happen. Also the oscillations around the target value afterwards should be avoided. In both the hypotension and CHF test case MAP does not reach the target value for MAP at all, but stays 8 or $11 \mathrm{mmHg}$ beneath. The controller needs more training to adapt correct dosages in these cases. To improve the controller, one idea is to switch the outputs to increment and decrement values, instead of set dosage values. This might improve the smoothness of the controlled signals.

Although the controller is already flexible in the sense that it can be adapted to different patients in choosing different target values during setup, while running regulation there is could still be learnt from past actions. The idea is to introduce some kind of online learning to create a personal controller, which can remember and learn the best treatment for each patient. Furthermore a comparative study is planned to compare the results of a fuzzy controller and a simple proportional-derivative controller in their performances for hemodynamic control. A final remark is, that the performance of the fuzzy controller is currently only verified in a canine simulation environment. It is expected, that real life experiments, will lead to further adaptations and improvements of the controller.

\section{ACKNOWLEDGMENT}

This research was funded by the Graduate School of Information Science in Health (GSISH) and the TUM Graduate School

\section{REFERENCES}

[1] S. Isaka and A. V. Sebald, "Control strategies for arterial blood pressure regulation." IEEE Trans Biomed Eng, vol. 40, no. 4, pp. 353-363, Apr 1993. [Online]. Available: http://dx.doi.org/10.1109/10.222328

[2] C. M. Held and R. J. Roy, "Hemodynamic management of congestive heart failure by means of a multiple mode rule-based control system using fuzzy logic." IEEE Trans Biomed Eng, vol. 47, no. 1, pp. 115-123, Jan 2000. [Online]. Available: http://dx.doi.org/10.1109/10.817626

[3] J. W. Huang and R. J. Roy, "Multiple-drug hemodynamic control using fuzzy decision theory." IEEE Trans Biomed Eng, vol. 45, no. 2, pp. 213228, Feb 1998. [Online]. Available: http://dx.doi.org/10.1109/10.661269

[4] Y. Gao and M. J. Er, "An intelligent adaptive control scheme for postsurgical blood pressure regulation." IEEE Trans Neural Netw, vol. 16, no. 2, pp. 475-483, Mar 2005. [Online]. Available: http://dx.doi.org/10.1109/TNN.2004.841798

[5] K. Kashihara, "Automatic regulation of hemodynamic variables in acute heart failure by a multiple adaptive predictive controller based on neural networks." Ann Biomed Eng, vol. 34, no. 12, pp. 1846-1869, Dec 2006. [Online]. Available: http://dx.doi.org/10.1007/s10439-006-9190-9

[6] P. of the International MultiConference of Engineers and C. S. . IMECS, Eds., Fuzzy Controller for Automatic Drug Infusion in Cardiac Patients, vol. 1. Hong Kong: M. Logesh Kumar, R.Harikumar, A. Keerthi Vasan, V.K.Sudhaman, March 2009.

[7] A. Mendoza Garcia, B. Baumgartner, U. Schreiber, M. Krane, A. Knoll, and R. Bauernschmitt, "Automedic: Fuzzy control development platform for a mobile heart-lung machine," in World Congress on Medical Physics and Biomedical Engineering, September 7 - 12, 2009, Munich, Germany, ser. IFMBE Proceedings, R. Magjarevic, O. Dssel, and W. C. Schlegel, Eds. Springer Berlin Heidelberg, 2009, vol. 25/7, pp. 685-688.

[8] M. A. E. Brawany, "A functional cardiovascular model with iv cardiac drugs action," The Online Journal on Electronics and Electrical Engineering, vol. 1, no. 1, 2009.

[9] C. Yu, R. J. Roy, and H. Kaufman, "A circulatory model for combined nitroprusside-dopamine therapy in acute heart failure." Med Prog Technol, vol. 16, no. 1-2, pp. 77-88, May 1990. 